\title{
Neonatal Diabetes Mellitus Due to KCNJ11 (KIR6.2) Mutation Successfully Treated with Sulfonylurea
}

Sehun Jang, MD, Misun Yang, MD, So Yoon Ahn, MD, PhD, Se In Sung, MD, PhD, Yun Sil Chang, MD, PhD, Won Soon Park, MD, PhD

Department of Pediatrics, Samsung Medical Center, Sungkyunkwan University School of Medicine, Seoul, Korea

\section{ABSTRACT}

Neonatal diabetes mellitus (NDM) is a rare disease that occurs at less than 6 months of age and is presumably caused by a mutation in the gene that affects pancreatic beta-cell function. Approximately $80 \%$ of NDM cases reveal a known genetic mutation, and mutations in potassium inwardly rectifying channel subfamily J member 11 (KCNJ11) and ABCC8 affecting the pancreatic beta-cell adenosine triphosphatesensitive potassium channel may be treated with oral sulfonylurea. Early recognition of mutations in $K C N J 11$ and $A B C C 8$ is important because early administration of sulfonylurea can not only control blood glucose levels but also improve neurodevelopmental outcomes. In the present study, we report a case of NDM that initially presented as diabetic ketoacidosis at the age of 1 month, accompanied by seizures during hospitalization. After confirmation of the KCNJ11 gene mutation (c.989A >C), we started administering oral sulfonylurea (glimepiride) at the age of 2 months. After gradually increasing the dosage of glimepiride, insulin was discontinued at the age of 3 months. To date, the infant's blood glucose levels have been well controlled without significant hypoglycemic events. No further episodes of seizures have occurred, and his developmental status is favorable.

Key Words: Diabetes mellitus, permanent neonatal; Kir6.2 channel; Sulfonylurea compounds; Glimepiride

\section{INTRODUCTION}

Neonatal diabetes mellitus (NDM) is defined as the presence of persistent hyperglycemia within the first 6 months of life. It often occurs at less than 6 months of age; however, it may also occur up to 12 months of age. It is presumably caused by a mutation in the gene that affects pancreatic beta-cell function. NDM occurs in approximately 1 in 90,000 to 160,000 live births ${ }^{1,2)}$. Approximately $80 \%$ of NDM cases are known to present a genetic mutation ${ }^{3)}$. Mutations in potassium inwardly rectifying channel subfamily J member 11 (KCNJ11) and affecting the pancreatic beta-cell adenosine triphosphate-sensitive potassium (K-ATP)
Received: 12 April 2021

Revised: 20 May 2021

Accepted: 24 May 2021

Correspondence to: Se In Sung, MD, $\mathrm{PhD}$

Department of Pediatrics, Samsung Medical Center, Sungkyunkwan University School of Medicine, 81 Irwonro, Gangnam-gu, Seoul 06351, Korea

Tel: +82-2-3410-1775

Fax: +82-2-3410-0043

E-mail: sein.sung@samsung.com

Copyright(c)

By Korean Society of Neonatology.

All right reserved.

This is an Open-Access article distributed under the terms of the Creative Commons At tribution Non-Commercial License (http:// creativecommons.org/licenses/by-nc/4.0), which permits unrestricted non-commercial use, distribution, and reproduction in any medium, provided the original work is pro perly cited. 
channel $(A B C C 8)$ may be treated with oral sulfonylurea and may account for approximately $40 \%$ of NDM cases. Early recognition of mutations in $K C N J 11$ and $A B C C 8$ via genetic testing is important so that sulfonylurea can be administered to control the blood glucose levels and improve neurodevelopmental outcomes $^{4)}$.

Herein, we report a case of NDM that initially presented as diabetic ketoacidosis (DKA) and seizures, confirmed by the KCNJ11 gene mutation. After genetic confirmation, the patient was suc cessfully treated with oral sulfonylurea.

\section{CASE REPORT}

A 34-day-old boy was admitted to the neonatal intensive care unit with fever, diarrhea, and vomiting. He was born at 40 weeks and 2 days of gestation and was delivered by cesarean section with a birth weight of 3,380 g. Initial vital signs revealed a blood pressure of $89 / 42 \mathrm{~mm} \mathrm{Hg}$, pulse rate of 148 beats/min, respiratory rate of 30 breaths $/ \mathrm{min}$, and body temperature of $38.2^{\circ} \mathrm{C}$. His body weight was $4.2 \mathrm{~kg}$ (25th to 50th percentile of growth curve). Upon examination, his mental status was alert without focal neurologic deficits; however, his general activity was decreased. His anterior fontanelle was isotense; however, skin mottling was observed on the whole body, and his tongue was dehydrated. His breathing sounds were clear, and his abdomen was soft. The results of initial laboratory test were as follows: whole blood glucose, $740 \mathrm{mg} /$ $\mathrm{dL}$; $\mathrm{pH}, 7.16$; partial pressure of $\mathrm{CO}_{2}, 21.0 \mathrm{~mm} \mathrm{Hg}$; bicarbonate, $7.6 \mathrm{mmol} / \mathrm{L}$; base excess, $-18.6 \mathrm{mmol} / \mathrm{L}$, sodium, $141 \mathrm{mmol} / \mathrm{L}$; potassium, $6.8 \mathrm{mmol} / \mathrm{L}$; chloride, $103 \mathrm{mmol} / \mathrm{L}$; beta hydroxybutyrate, $3.5 \mathrm{mmol} / \mathrm{L}$ (reference range, 0 to 0.6 ); C-peptide, $0.49 \mathrm{ng} /$ $\mathrm{mL}$ (reference range, 1.06 to 3.53); insulin, $4.0 \mu \mathrm{IU} / \mathrm{mL}$ (reference range, 1.1 to 11.6); urine glucose, $3+$; urine ketone, 3+. Microbiologic evaluations, including blood culture, cerebrospinal fluid culture, norovirus antigen, rotavirus antigen, and respiratory virus polymerase chain reaction, were all negative.

Massive fluid resuscitation was performed, and regular insulin was administered via continuous infusion. Blood glucose gradu ally decreased to $420 \mathrm{mg} / \mathrm{dL}$ on the second day of hospitalization. After the third day of hospitalization, blood glucose was main tained in the range of 150 to $330 \mathrm{mg} / \mathrm{dL}$. During the first and second days of hospitalization, he presented with generalized tonic-clonic seizures; hence, we administered intravenous phenobarbital. At that time, no hypoglycemia or electrolyte imbalance was observed. Electroencephalography revealed ictal waves in the left hemisphere; however, brain ultrasonography revealed no structural abnormalities. After the dehydration improved and blood glucose levels were controlled, continuous infusion of insulin was tapered, and subcutaneous intermediate-acting insulin (neutral protamine Hagedorn) was administered.

To differentiate type 1 diabetes mellitus (DM), we tested insulin autoantibodies, pancreatic islet cell antibodies, and anti-glutamic acid decarboxylase II antibodies; all these tests revealed negative results. Abdominal ultrasonography revealed no abnormalities in the pancreatic structure. The pancreatic elastase level in the stool, reflecting the exocrine function of the pancreas, was within normal limits. To diagnose genetic neonatal diabetes, we tested the chromosomal microarray for 6q24 anomalies and performed a KCNJ11/ABCC8gene analysis. Direct sequencing of the KCNJ11 gene, encoding the Kir6.2 subunit of the K-ATP channel, confirmed the presence of a mutation (c.989A >C) (Figure 1). ABCC8 mutation was not detected, and no microdeletion or duplication was observed on the chromosome microarray.

After confirmation of the KCNJ11 mutation, we started administering $0.1 \mathrm{mg} / \mathrm{kg} /$ day oral sulfonylurea (glimepiride) and gradually increased the dosage, thereby tapering the insulin dosage. Before initiating the sulfonylurea therapy, his daily insulin re quirement was up to $1.58 \mathrm{U} / \mathrm{kg} /$ day. After 37 days, $1.06 \mathrm{mg} / \mathrm{kg} /$

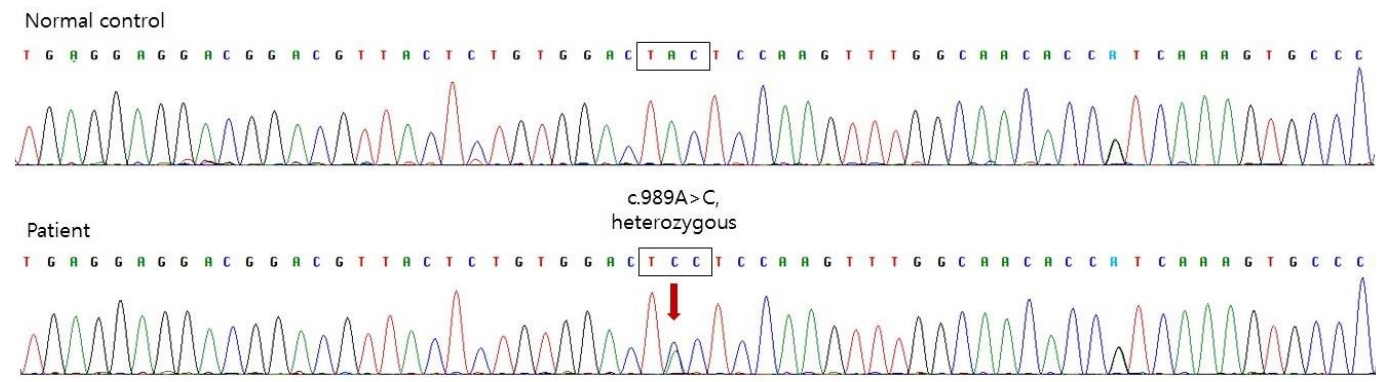

Figure 1. Direct sequencing of the potassium inwardly rectifying channel subfamily J member 11 $($ KCNJ11) gene revealed a heterozygous mutation of c.989A $>$ C (p.Tyr330Ser). 
day glimepiride was administered and insulin was discontinued. Even after discontinuing insulin injection, his blood glucose level was well controlled and glimepiride requirement gradually decreased, with the C-peptide level maintained in the normal range (Figure 2).

The patient is now 15 months old, with a body weight of $11.7 \mathrm{~kg}$ (90 percentile of the growth curve). He can walk alone, perform the pincer grasp, and follow simple commands. His blood glucose level was well controlled, without significant hypoglycemic events. His current glimepiride dosage is $0.1 \mathrm{mg} / \mathrm{kg} /$ day, which is significantly lower than the usual maintenance dose of glimepiride $(0.3$ to $0.8 \mathrm{mg} / \mathrm{kg} /$ day). To date, no further episodes of seizures have occurred.

\section{DISCUSSION}

DM diagnosed after 6 months of age is most likely to be type 1 DM with an autoimmune cause. In contrast, in NDM, more than $80 \%$ of the cases are caused by genetic mutations ${ }^{3)}$. It is essential to detect genetic mutations in patients with NDM for appropriate treatment options and to predict the disease prognosis. In a large international cohort study, 93\% of patients with the $K C N J 11 \mathrm{mu}$ tation maintained excellent glycemic control with sulfonylurea for a long-term period ${ }^{5)}$. The most important factor affecting sulfonylurea responsiveness is the age at which sulfonylurea treat ment is administered. In a mouse model, chronic hyperglycemia is known to impair beta-cell function and reduce beta-cell mass ${ }^{6)}$.
Therefore, if diagnosed early, the patient's beta-cells would be well preserved because it has been exposed to chronic hypergly. cemia for a shorter period, and it could respond to sulfonylurea ${ }^{7)}$. This result emphasizes the importance of early genetic diagnosis of KCNJ11-related neonatal diabetes. Mutated K-ATP channels are also expressed in neural tissues and cause neurological manifestations, such as developmental delays and epilepsy, which is known as the developmental delay, epilepsy, and neonatal diabetes (DEND) syndrome. Sulfonylurea treatment has been shown to be effective in improving neurological manifestations of the DEND syndrome ${ }^{4)}$.

Sulfonylurea treatment is well tolerated by most patients because of its mild side effects. Common side effects of sulfonylureas include hypoglycemia and digestive problems (diarrhea and nausea). Most side effects are known to be mild and have never interrupted treatment ${ }^{8)}$. Hypoglycemia was reported monthly or less frequently in most patients, and hypoglycemic episodes did not provoke seizures or unconsciousness ${ }^{9)}$.

By far, in South Korea, a total of six cases of NDM due to the KCNJ11 mutation have been reported. The mean age at diagnosis of diabetes was 6.5 weeks (range, 3 to 9) and the mean age of in itiating sulfonylurea treatment was 8.5 years (range, 0.3 to 18$)^{10-14)}$. Of these six patients, the five cases in which sulfonylurea treatment was initiated at a younger age, that is from 0.3 to 17 years, were successfully switched from insulin to sulfonylurea treatment. One patient who received sulfonylurea treatment at 18 years failed to switch from insulin to sulfonylurea ${ }^{11)}$. In the present case, the patient presented with severe DKA and seizures, raising concerns

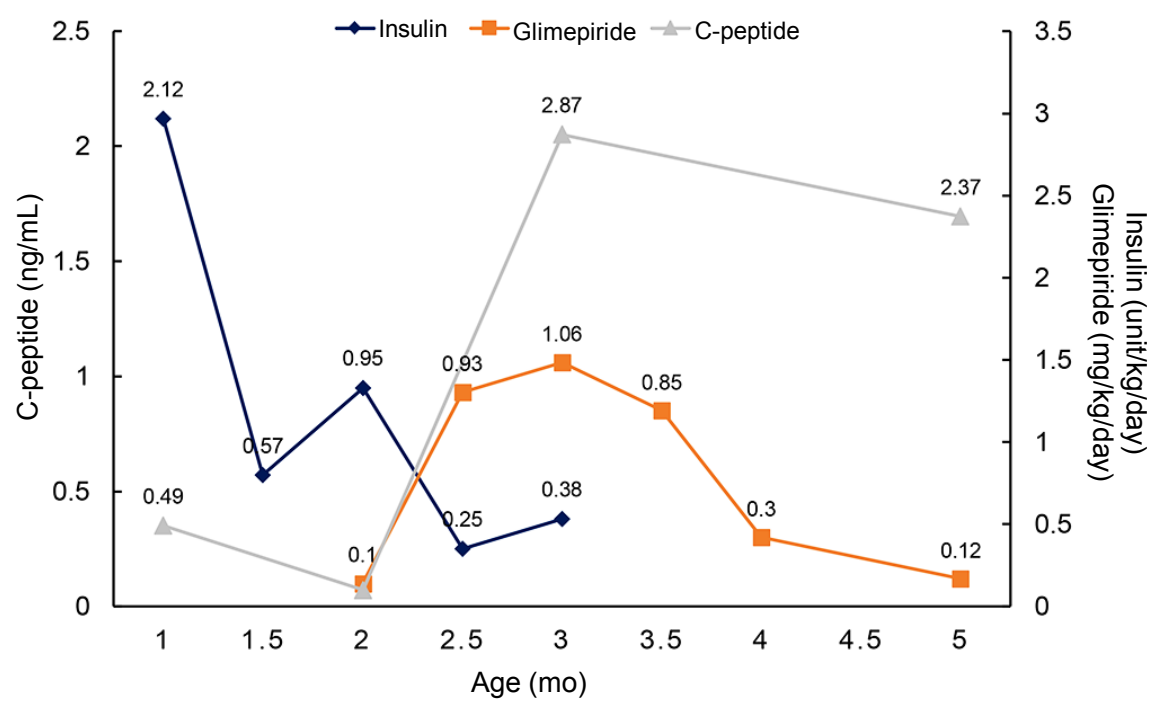

Figure 2. The clinical course of the present case. After initiation of sulfonylurea treatment, insulin was discontinued and the $\mathrm{C}$ - peptide level was maintained in the normal range. 
for the DEND syndrome. However, due to early detection of the KCNJ11 mutation, sulfonylurea treatment could be initiated at the age of 2 months, the earliest age reported in Korea. Early sulfonylurea treatment led to a favorable treatment response.

It remains controversial whether sulfonylurea treatment should be attempted immediately or after genetic confirmation in patients with neonatal diabetes. In a recent study by Carmody et al. ${ }^{15)}$, empirical sulfonylurea therapy appeared to be safe and often successful in patients with neonatal diabetes even before receiving the genetic test results, with potential beneficial effects of sulfonylurea on glycemic control and neurodevelopmental outcomes. However, in a case report by Yildiz et al. ${ }^{16)}$, empirical sulfonylurea therapy did not improve glycemic control and even worsened it due to emerging insulin resistance. The patient was proven to have a mutation in the INS gene encoding insulin and its precursors, which is generally best treated with insulin alone. Therefore, it is considered safe to initiate sulfonylurea therapy after confirmation of the mutation.

In conclusion, early genetic testing is important in patients with diabetes before 6 months of age because it can help predict responsiveness to oral sulfonylurea. Early administration of sulfonylurea in certain genotypes is beneficial for glycemic control and neurodevelopmental outcomes.

\section{ARTICLE INFORMATION}

\section{Ethical statement}

This study was approved by the Institutional Review Board of Samsung Medical Center (IRB No. 2021-03-051). Written informed consent was obtained from the the parents of the patient.

\section{Conflicts of interest}

No potential conflict of interest relevant to this article was reported.

\section{Author contributions}

Conception or design: S.J., S.I.S.

Acquisition, analysis, or interpretation of data: S.J., M.Y., S.Y.A., S.I.S.

Drafting the work or revising: S.J., M.Y., S.Y.A., S.I.S., Y.S.C., W.S.P.

Final approval of the manuscript: S.J., S.I.S., Y.S.C., W.S.P.

\section{ORCID}

Sehun Jang https://orcid.org/0000-0002-2841-0648

Se In Sung https://orcid.org/0000-0002-8717-6142

\section{Acknowledgments}

None

\section{REFERENCES}

1. Letourneau LR, Carmody D, Wroblewski K, Denson AM, Sanyoura M, Naylor RN, et al. Diabetes presentation in infancy: high risk of diabetic ketoacidosis. Diabetes Care 2017;40:e147-8.

2. Nansseu JR, Ngo-Um SS, Balti EV. Incidence, prevalence and genetic determinants of neonatal diabetes mellitus: a systematic review and meta-analysis protocol. Syst Rev 2016;5:188.

3. De Franco E, Flanagan SE, Houghton JA, Lango Allen H, Mackay DJ, Temple IK, et al. The effect of early, comprehensive genomic testing on clinical care in neonatal diabetes: an international cohort study. Lancet 2015;386:957-63.

4. Shah RP, Spruyt K, Kragie BC, Greeley SA, Msall ME. Visuomotor performance in KCNJ11-related neonatal diabetes is impaired in children with DEND-associated mutations and may be improved by early treatment with sulfonylureas. Diabetes Care 2012;35:2086-8.

5. Bowman P, Sulen A, Barbetti F, Beltrand J, Svalastoga P, Codner E, et al. Effectiveness and safety of long-term treatment with sulfonylureas in patients with neonatal diabetes due to KCNJ11 mutations: an international cohort study. Lancet Diabetes Endocrinol 2018;6:637-46.

6. Brereton MF, Rohm M, Shimomura K, Holland C, TornovskyBabeay S, Dadon D, et al. Hyperglycaemia induces metabolic dysfunction and glycogen accumulation in pancreatic $\beta$-cells. Nat Commun 2016;7:13496.

7. Ashcroft FM, Puljung MC, Vedovato N. Neonatal diabetes and the KATP channel: from mutation to therapy. Trends Endocrinol Metab 2017;28:377-87.

8. Garcin L, Mericq V, Fauret-Amsellem AL, Cave H, Polak M, Bel trand J. Neonatal diabetes due to potassium channel mutation: response to sulfonylurea according to the genotype. Pediatr Diabetes 2020;21:932-41.

9. Lanning MS, Carmody D, Szczerbinski L, Letourneau LR, Naylor RN, GreeleySAW.Hypoglycemia in sulfonylurea-treated KCNJ11 -neonatal diabetes: mild-moderate symptomatic episodes occur infrequently but none involving unconsciousness or seizures. Pediatr Diabetes 2018;19:393-7.

10. Kim MS, Kim SY, Kim GH, Yoo HW, Lee DW, Lee DY. Sulfonylurea therapy in two Korean patients with insulin-treated neonatal diabetes due to heterozygous mutations of the KCNJ11 gene en- 
coding Kir6.2. J Korean Med Sci 2007;22:616-20.

11. Heo JW, Kim SW, Cho EH. Unsuccessful switch from insulin to sulfonylurea therapy in permanent neonatal diabetes mellitus due to an R201H mutation in the KCNJ11 gene: a case report. Diabetes Res Clin Pract 2013;100:e1-2.

12. Ahn SY, Kim GH, Yoo HW. Successful sulfonylurea treatment in a patient with permanent neonatal diabetes mellitus with a novel KCNJ11 mutation. Korean J Pediatr 2015;58:309-12.

13. Cho JH, Kang E, Lee BH, Kim GH, Choi JH, Yoo HW. DEND syndrome with heterozygous KCNJ11 mutation successfully treated with sulfonylurea. J Korean Med Sci 2017;32:1042-5.

14. Yoon JS, Park KJ, Sohn YB, Lee HS, Hwang JS. Successful swit- ching from insulin to sulfonylurea in a 3-month-old infant with diabetes due to p.G53D mutation in KCNJ11. Ann Pediatr Endocrinol Metab 2018;23:154-7.

15. Carmody D, Bell CD, Hwang JL, Dickens JT, Sima DI, Felipe DL, et al. Sulfonylurea treatment before genetic testing in neonatal diabetes: pros and cons. J Clin Endocrinol Metab 2014;99:E270914.

16. Yildiz M, Akcay T, Aydin B, Akgun A, Dogan BB, De Franco E, et al. Emergence of insulin resistance following empirical glibenclamide therapy: a case report of neonatal diabetes with a recessive INS gene mutation. J Pediatr Endocrinol Metab 2018;31: 345-8. 